Revue d'histoire de l'Amérique française

6. REVUE D.HISTOIRE DE L'AMÉRIQUE FRANÇAISE

\title{
La rente du faubourg Saint-Roch à Québec - 1750-1850
}

\section{Louise Dechêne}

Volume 34, numéro 4, mars 1981

URI : https://id.erudit.org/iderudit/303905ar

DOI : https://doi.org/10.7202/303905ar

Aller au sommaire du numéro

Éditeur(s)

Institut d'histoire de l'Amérique française

ISSN

0035-2357 (imprimé)

1492-1383 (numérique)

Découvrir la revue

Citer cet article

Dechêne, L. (1981). La rente du faubourg Saint-Roch à Québec — 1750-1850.

Revue d'histoire de l'Amérique française, 34(4), 569-596.

https://doi.org/10.7202/303905ar d'utilisation que vous pouvez consulter en ligne.

https://apropos.erudit.org/fr/usagers/politique-dutilisation/ 


\title{
LA RENTE DU FAUBOURG SAINT-ROCH À QUÉBEC - 1750-1850
}

\author{
LOUISE DECHÊNE \\ Département d'histoire \\ Université McGill
}

Cette étude est d'abord une contribution à l'histoire des villes canadiennes des XVIIIe et XIXe siècles. Le terrain d'enquête étant encore relativement neuf, nous n'avons pas pu, comme nous l'aurions souhaité, établir des comparaisons avec d'autres villes, d'autres quartiers. Le problème demeure donc posé: les conditions du lotissement de Saint-Roch sont-elles exceptionnelles ou communément répandues dans la province à la même époque?

Malgré son caractère circonscrit, cette recherche sur la propriété foncière soulève des problèmes plus généraux. Dans la longue période de transition qui précède l'industrialisation du pays vers la fin du XIXe siècle, les historiens et les économistes ont généralement perçu la réalité économique sous la forme d'une dualité. D'une part, le commerce extérieur axé sur l'exportation en Grande-Bretagne des grands «staples», fourrures, bois et blé du Haut-Canada, secteur dynamique et seule source d'accumulation; d'autre part, le système de production et de reproduction local, faible, bloqué par les règles du droit coutumier, décourageant les investissements, lequel ne jouerait à peu près aucun rôle dans le développement économique. Sans nier l'effet d'entraînement du grand commerce, il nous apparaît nécessaire d'étudier les liaisons qui existent entre les deux secteurs que néglige la démarche dichotomique. Diverses formes d'accumulation primitive, étroitement imbriquées, se déploient durant cette phase séculaire. Il n'est pas juste, par exemple, de prétendre que la seigneurie s'est maintenue jusqu'en 1854 contre la volonté des marchands, simplement parce que l'élite canadienne-française s'y accrochait pour des raisons de prestige et de stratégie politique. Loin d'être un repoussoir, les diverses ponctions et contraintes extra-économiques autorisées par [569] 
PLAN DE LA VILLE DE CIIEIEC 1829 (J. Hame1)

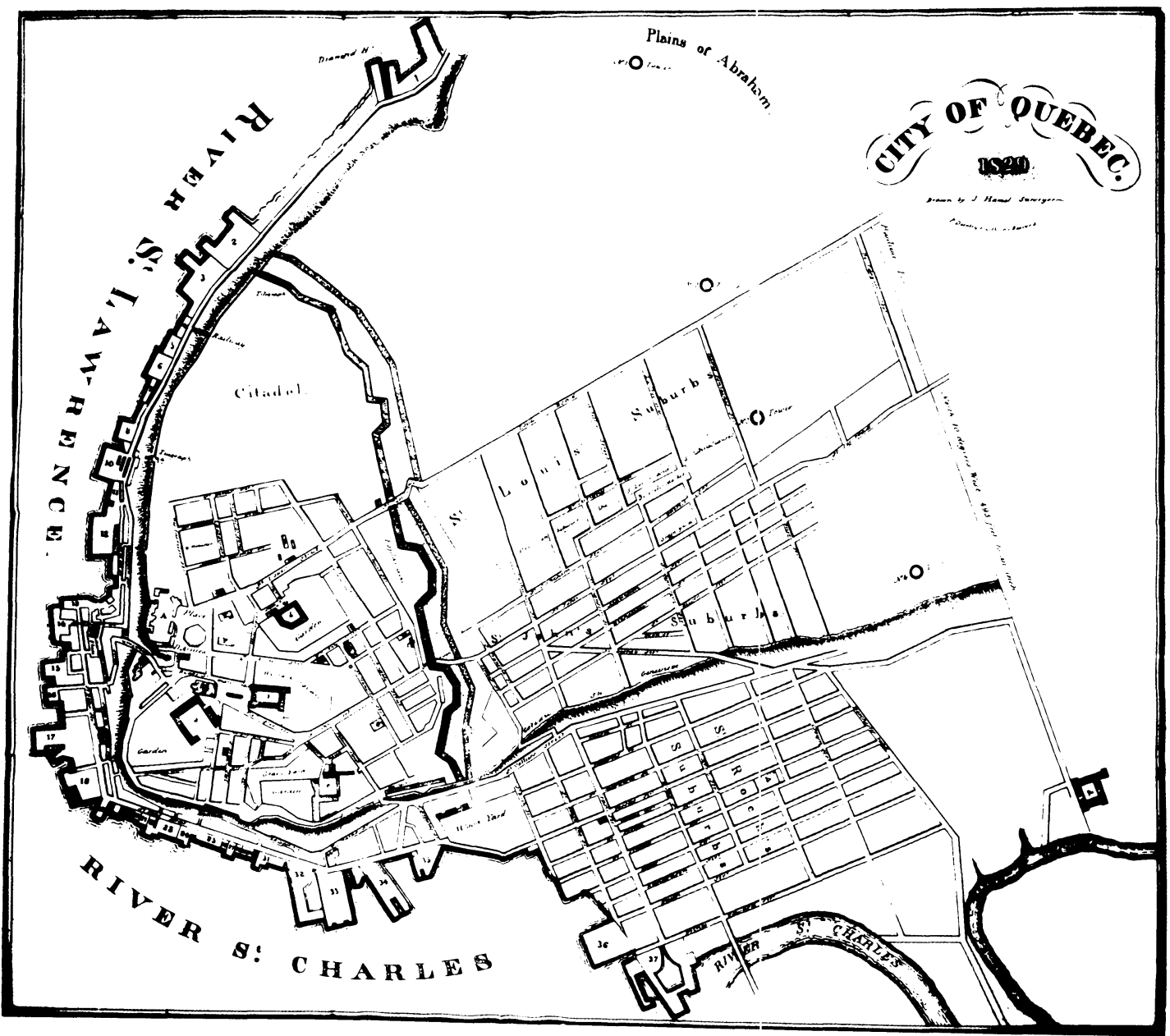


la loi des fiefs constituaient une forme idéale de prélèvement sur la petite production. La bourgeoisie de toutes origines trouvait provisoirement son compte à maintenir les rapports sociaux existants, un type de propriété qui n'entre pas en contradiction avec le développement général du pays avant la seconde moitié du XIXe siècle. Saint-Roch représente un bon exemple de la vigueur d'une institution qui offre aux lotisseurs urbains des moyens de contrôle dont ils ont pu mesurer l'efficacité en milieu rural.

Le cas de Saint-Roch nous invite en même temps à réfléchir sur les conditions qui déterminent la répartition des actifs des fortunes bourgeoises et autres et à renoncer au psychologisme stérile. Nous avons observé un groupe de négociants-entrepreneurs qui immobilisent une partie de leur patrimoine dans des rentes foncières et constituées, soit une forme traditionnelle de placement. Plutôt que de parler de choix, inspiré par la mentalité individuelle de ces détenteurs de capitaux, ce sont les contraintes auxquelles ils sont assujettis qu'il faut mettre au premier plan et nous retiendrons que, jusque vers 1840, les épargnants du Bas-Canada sont forcés de fonctionner dans un espace économique réduit où les marges de décisions sont infimes.

\section{I - LA FORMATION DU FAUBOURG}

Fondé au début du XVIIe siècle, Québec compte environ 5000 habitants en 1750 et 50000 un siècle plus tard. La ville comprend deux quartiers: la Basse-Ville formée par une longue et étroite bande de terre qui contourne le pied de la falaise et la Haute-Ville, juchée sur cet escarpement de cent mètres et retranchée de la campagne derrière par ses remparts. Deux côtes abruptes lient l'une et l'autre. La propriété administrative, militaire et religieuse est concentrée à la Haute-Ville et les marchands sont installés à la Basse-Ville, autour du marché. La topographie sociale se transforme à partir de la fin du XVIIIe siècle, avec la naissance des deux faubourgs Saint-Roch et Saint-Jean, le regroupement de la bourgeoisie à la Haute-Ville et l'entassement des travailleurs du port dans des maisons de rapport à la Basse-Ville ${ }^{1}$.

1 Pour une introduction utile à l'histoire de Québec, voir Edward H. Dahl et coll., $L a$ ville de Québec 1800-1850: un inventaire de cartes et plans (Ottawa, Musée national de l'homme, 1975). 
Dans les débuts, quelques privilégiés se partagèrent les terres autour de la ville. C'est ainsi que la vallée de la rivière SaintCharles fut concédée d'est en ouest à Louis Hébert, un des premiers colons à s'établir à demeure dans le pays ${ }^{2}$, aux Jésuites, à l'Hôtel-Dieu et à l'Hôpital-Général. À la faveur de son mariage avec une descendante de Hébert, le négociant Charles Aubert de La Chesnaye acquit la terre dite de la Pointe-aux-Lièvres, de 86 arpents [29,4 ha] de superficie, et y fit construire sa résidence appelée La Maison Blanche. Mise aux enchères en 1720, la terre fut adjugée à Henri Hiché, alors commis aux magasins du roi. Hiché, qui avait fait un mariage avantageux, poursuivit une carrière rapide: notaire en 1725, procureur à la Prévôté en 1736 et conseiller au Conseil Supérieur de la colonie en $1754^{3}$. Il demeurait à la Maison Blanche, surveillant ses métayers, en attendant le moment propice pour procéder au lotissement, ce qui ne pouvait tarder car les grandes réserves ecclésiastiques limitaient l'espace à construire à l'intérieur de la ville.

Les premières ventes de terrains remontent à 1731 , mais le mouvement ne commence à prendre de l'ampleur que vingt ans plus tard, avec 33 aliénations en 1751, 20 et 22 au cours des deux années suivantes, après quoi la guerre réduit la demande ${ }^{4}$. La terre de Hiché est dans la censive du roi ${ }^{5}$ et grâce à la réfection du papier-terrier du domaine royal nous avons une bonne image de l'occupation du nouveau quartier à la fin du régime français. Les 118 propriétaires sont pressés dans l'angle sud-est, le plus rapproché du Palais de l'Intendance, ainsi que le long de la rue SaintVallier, dite des Tanneurs. Une chapelle dédiée à Saint-Roch, que les Récollets avaient fait élever le long du rivage, donne son nom au faubourg qui, dès les débuts, présente un caractère nettement populaire.

2 Le titre primitif qui daterait de 1626 n'a pas été retrouvé. Rapport des archives canadiennes (1884); 26.

3 Il épousa en 1713 Marguerite Le Gardeur qui appartenait à une des familles les plus puissantes de la colonie. P.-G. Roy, La famille Hiché (Lévis, 1935).

4 Le mouvement de ce premier lotissement a été reconstitué à partir du greffe du notaire Lanouillier, ANQ [Archives nationales du Québec] et du papier-terrier du domaine royal de 1758: ANQ, NF8.

5 Plusieurs petits fiefs furent concédés dans la ville et la banlieue mais le Roi conserva la seigneurie directe sur le reste de ce territoire. Les terrains sont chargés de cens portant lods et ventes, amendes, etc., payables au domaine royal. Sur la terre de Hiché, la redevance annuelle est fixée uniformément à $5 \mathrm{~s}$. $6 \mathrm{~d}$. pour chaque censitaire. 
TABLEAU 1

POPULATION ET NOMBRE DE MAISONS DANS

LE FAUBOURG SAINT-ROCH

1744-1831

$\begin{array}{rcc}\text { Année } & \text { Population } & \text { Nombre de maisons } \\ 1744 & 245 & \\ 1795 & 829 & \\ 1805 & 1497 & 526 \\ 1815 & 3821 & 609 \\ 1818 & 4611 & 804 \\ 1824 & 5800 & \\ 1825 & 6307 & 1137 \\ 1831 & 7903 & \end{array}$

Sources: Recensement de 1744, dans le Rapport des Archives du Québec (1939-40); les recensements du curé de Québec pour 1795, 1805, 1815, 1818, 1824 et 1825 dans Ibid (1948-49) et dans H. Provost, Le recensement de la ville de Québec en 1818 par le curé Jos. Signay (Québec, 1976) et les chiffres cités par E.H. Dahl et coll., op. cit.; le recensement de 1831 dans Appendice des Journaux de l'Assemblée législative, vol. 41.

Entre la fin de la guerre et 1795 , la population de Saint-Roch croît relativement lentement. Compte tenu de la destruction massive par les bombardements, il s'agirait d'abord d'un mouvement de repeuplement. Il y a un premier élan entre 1795 et 1805 , suivi d'un second beaucoup plus fort dans la décennie suivante. Nous savons d'autre part que l'occupation atteint presque les limites de la terre de la Pointe-aux-Lièvres en 1815 , ce qui explique le ralentissement subséquent. Ce n'est que lorsque la ferme voisine de la Vacherie, ancienne propriété des Jésuites, est lotie à son tour, vers 1825 , que le mouvement peut reprendre de l'amplitude. Le faubourg va dès lors se prolonger au-delà de ses frontières primitives, effacées par de nouvelles divisions administratives ${ }^{6}$. L'augmentation du nombre de maisons suit à peu près celle de la population, ce qui indique un même type de développement domiciliaire tout au long de la période.

${ }^{6}$ Antonio Drolet, La ville de Québec. Historique municipale (1833-1867). La Société historique de Québec 1967, III: 121ss. 
Les autorités municipales n'interviennent pas dans l'organisation du quartier. Les propriétaires du fonds ont la haute main sur le lotissement et le tracé des rues. Celles-ci, non pavées, entretenues par les riverains, n'ont que $4,8 \mathrm{~m}$. de large, ce qui est bien inférieur aux standards de l'époque 7 . Il n'y a aucun service d'éclairage, d'aqueduc et d'égoûts, mais ces derniers traits ne sont pas spécifiques de Saint-Roch. Il faut attendre les épidémies meurtrières, les grands incendies et les remaniements administratifs de la seconde moitié du XIXe siècle pour que les pouvoirs publics se préoccupent de ces questions. Les autorités religieuses ne sont guère plus empressées. Ce n'est qu'après quelque soixante ans d'occupation que la première église est bâtie et il ne semble pas y avoir eu de prêtre résident avant $1818^{8}$.

La grande cour à bois du roi établie dès le régime français dans l'angle du Palais et de la rue Saint-Roch bloquait déjà les voies de sortie du faubourg. À partir de 1807, les terrains le long de la rivière Saint-Charles se couvrent de chantiers et de cours à bois privées et, en 1810, le gouvernement permet aux marchands d'occuper tout le littoral de la rivière et de son estuaire, y compris les terres immergées à marée haute ${ }^{9}$. Du coup le faubourg se retrouve littéralement emmuré au nord et à l'est comme il l'était du côté sud par le côteau quasi vertical. À plusieurs reprises les citoyens protestent contre cet enfermement, réclament un marché et des chemins carossables pour communiquer avec la ville. Une requête de 1830 souligne que les femmes mettent plus de deux heures à atteindre le marché de la Basse-Ville à travers les chantiers boueux, quand elles ne risquent pas de se geler les membres en hiver ${ }^{10}$.

Le faubourg se présente comme un tissu serré et assez régulier d'îlots rectangulaires. Dans la plupart de ceux-ci, les emplacements alignés de part et d'autre d'une ligne médiane adoptent à peu près la forme d'un carré de 50 pieds de côté ${ }^{11}$; ailleurs, ils perdent en fa-

7 Antonio Drolet, op. cit., III: 33 et II: 70 et 83. Les règlements en vigueur dans les villes de la colonie imposaient 30 pieds ou $9,7 \mathrm{~m}$. dès le XVIIe siècle.

8 Archives du diocèse de Québec, vol. H, 217ss: règlements et lettres du chapelain (1818). Saint-Roch est érigé en paroisse en 1829.

9 Concession du 4 oct. 1810 à J. Richardson de 537166 pieds carrés, APC [Archives publiques du Canada], RG1, L3L, fo 47680-1 et le plan, fo 47684. Nous tenons à remercier Claudette Lacelle qui nous a signalé l'existence de plusieurs documents sur Saint-Roch aux Archives publiques.

10 Drolet, op. cit., II: 112-115.

11 Tout au long de la période étudiée, c'est le «pied français», mesure de Paris [0,324 m.] qui sert d'unité et nous avons préféré le conserver dans cette étude afin de suivre plus facilement l'évolution des prix et des rentes. 
çade ce qu'ils gagnent en profondeur et nous retiendrons une superficie moyenne de 2500 pieds carrés $\left[262,4 \mathrm{~m}^{2}\right]$ pour les emplacements vendus au XIXe siècle. Auparavant et particulièrement le long de la rue Saint-Vallier, qui serpente le long du côteau, le lotissement était moins régulier, variant entre 1000 et 6000 pieds carrés. La construction est l'affaire des propriétaires individuels. Ce sont des maisons basses d'environ 625 pieds $^{2}$ avec un étage en comble au-dessus du rez-de-chaussée, et presque toutes sont en bois ${ }^{12}$. On compte généralement deux maisons juxtaposées sur la façade d'un même emplacement, ce qui laisse encore assez d'espace derrière pour bâtir des ateliers, hangars, étables ou écuries. Le recensement de 1831 dénombre 216 chevaux, 177 vaches, 26 moutons et 236 cochons dans le faubourg ${ }^{13}$. Lorsque le quartier est rasé par le feu en 1845, les pertes sont évaluées à 1630 maisons et 3000 boutiques et hangars, rapport qui témoigne de l'encombrement dans les arrière-cours ${ }^{14}$.

Pour faire la ventilation de la propriété entre les diverses catégories professionnelles, nous nous sommes servie du rôle d'évaluation de 1821 , le premier qui ait été conservé ${ }^{15}$. Il offre l'avantage, d'une part, de représenter le faubourg au moment où celui-ci est encore circonscrit dans ses frontières originelles et, d'autre part, de distinguer les maisons occupées par leur propriétaire, des autres. Mais le document est bâclé et le mélange d'anglais et de français dans la nomenclature des professions rend même la lecture difficile.

12 Ces observations reposent sur l'analyse de quelque 400 marchés de construction passés entre 1800 et 1844 que nous avons pu faire grâce au précieux relevé de G.-G. Bastien et collaborateurs, Inventaire des marchés de construction passés à Québec entre 1800 et 1870, 3 vols (Ottawa, 1974). Voir aussi l'article de Jacques Bernier, «La construction domiciliaire à Québec, 1810-1820» dans la Revue d'histoire de l'Amérique française [RHAF], 31,4 (mars 1978): 550-552. Les ouvriers de Saint-Roch construisent souvent leur maison eux-mêmes mais, le cas échéant, passent quand même un contrat pour la maçonnerie des fondations et la cheminée.

13 Recensement du Bas-Canada pour l'année 1831, Appendice des Journaux de l'Assemblée législative, vol. 41.

14 Drolet, op. cit., vol. III, 66.

15 La taxe municipale fut établie en 1795. Drolet, op. cit., vol. II, 132-133. 
TABLEAU 2

RÉPARTITION DE LA PROPRIÉTÉ

DU FAUBOURG SAINT-ROCH

PAR GROUPES PROFESSIONNELS EN 1821

\begin{tabular}{|l|r|r|r|r|r|}
\hline \multirow{2}{*}{ Professions } & \multicolumn{3}{|c|}{ Propriétaires } & \multirow{2}{*}{ Locataires } & \multirow{2}{*}{ Ensemble } \\
\cline { 2 - 5 } & Occupants & $\begin{array}{c}\text { Non- } \\
\text { occupants }\end{array}$ & Total & & \\
\hline Professions libérales & 1 & 5 & 6 & - & 6 \\
Négociants, constructeurs de navires & 3 & 4 & 7 & - & 7 \\
Commerçants, commis & 52 & 27 & 79 & 61 & 140 \\
Ouvriers du bâtiment & 158 & 27 & 185 & 76 & 261 \\
Autres hommes de métier & 100 & 25 & 125 & 60 & 185 \\
Chartiers, navigateurs & 39 & 6 & 45 & 24 & 69 \\
Domestiques, divers & 3 & 3 & 6 & 16 & 22 \\
Journaliers & 49 & 8 & 57 & 124 & 181 \\
Bourgeois, veuves et héritiers & 41 & 16 & 57 & - & 57 \\
Cultivateurs & 4 & 16 & 20 & - & 20 \\
Profession inconnue & 23 & 51 & 74 & 107 & 181 \\
TOTAI & 473 & 188 & 661 & 168 & 1129 \\
\hline
\end{tabular}

Source: Rôle d'évaluation municipale, Archives de l'Hôtel de Ville.

Les propriétaires sont imposés au prorata de la valeur locative de l'immeuble et les locataires sont sujets à une corvée, évaluée uniformément à 2 shillings 6 pence ${ }^{16}$ et acquittée généralement en argent. Mais qui sont exactement ceux que le rôle désigne comme locataires? Il y a tout lieu de croire que la catégorie comprend tous les hommes du quartier non imposés pour une propriété ${ }^{17}$. Si c'est le cas, nous avons affaire ici non seulement à des chefs de ménage locataires d'un logement, mais à un grand nombre d'individus actifs qui partagent le logement du propriétaire, tels que compagnons et pensionnaires. La prépondérance des ménages de propriétaires serait donc supérieure à ce qui apparaît dans le tableau.

La catégorie des propriétaires non-occupants soulève un second problème. Les nombres que nous donnons ici ont été obte-

16 Après 1760, on utilise deux systèmes de monnaie de compte dans le Bas-Canada: la livre française (1.) et la livre «courante» (£), qui compte 20 shillings (sh.) et 240 pence (d.). La conversion d'un système à l'autre est sur le pied de $£ 1=241$. Notons que le système comptable britannique sert pour les opérations commerciales ordinaires tandis que le système français est utilisé surtout pour les transactions foncières, le calcul des rentes d'origine féodale particulièrement. La distinction est assez significative pour ne pas l'effacer par des conversions systématiques.

17 C'est ainsi que le règlement du 31 mars 1843 sur les taxes municipales définit la catégorie et on peut penser que tel était l'usage antérieurement. Archives de l'Hôtel de Ville, rôle d'évaluation, microfilm no 6. 
nus après jumelage des individus qui possèdent plus d'une maison. S'il s'agit d'un occupant, il est compté une seule fois, comme tel. Cinquante-quatre propriétaires demeurant à Saint-Roch, ou $11,4 \%$ de la catégorie, sont dans ce cas, possédant, pour la majorité d'entre eux, deux maisons voisines ${ }^{18}$. Après cette opération, il reste néanmoins 188 propriétaires qui selon les apparences ne demeurent pas à Saint-Roch. Les immeubles du faubourg intéresseraient-ils la bourgeoisie de la ville? Plusieurs indices démentent cette hypothèse: d'abord, aucune concentration de propriétés dans cette catégorie puisque $88 \%$ ne possèdent qu'une seule maison; en second lieu, l'éventail des professions, voire même des patronymes, est sensiblement le même que chez les occupants; enfin les listes des débits-rentiers que nous avons analysées ${ }^{19}$ ne renferment essentiellement que des habitants du faubourg. D'où une seconde hypothèse beaucoup plus plausible: bon nombre de ces absents, personnes âgées surtout, seraient des gens du faubourg qui louent leur maison pour aller vivre en pension, chez leurs enfants par exemple. Quoi qu'il en soit, la petite propriété est certainement le trait dominant à Saint-Roch et les indices permettent de supposer que ces ménages d'artisans sont volontiers élargis par la présence de compagnons, parents et pensionnaires.

La valeur locative de ces propriétés est dans l'ensemble extrêmement faible, soit $£ 11$ [264 1.] et les deux tiers des immeubles se situent au-dessous de cette moyenne. A titre de comparaison, notons que la moyenne la plus basse observée dans un quartier ouvrier de Montréal à la même époque est de $£ 16^{20}$ ou encore, que la valeur locative d'une maison en pierres, mais modeste, dans la ville de Québec est d'environ $£ 50$. Or, comme nous avons pu le vérifier ${ }^{21}$, la valeur portée au rôle n'est pas fantaisiste mais correspond assez étroitement au loyer que le propriétaire peut tirer de l'immeuble.

Saint-Roch est aussi le lieu de travail de la très grande majorité de cette population, encore formée en 1821 d'artisans établis à leur compte. La Canada Tannery House semble être la seule manu-

18 Douze individus possèdent plus de deux maisons, soit un charron (6), un charpentier et un perruquier (5), des maçons, menuisiers et commerçants (3 et 4).

19 Voir infra, tableau 5.

20 Soit le faubourg Sainte-Marie dit Québec. Voir P.-A. Linteau et J.-C. Robert, «Propriété foncière et société à Montréal: une hypothèse», RHAF 28, 1 (juin 1974): 52.

21 En comparant le montant des baux passés devant notaire par George Pozer avec la valeur locative des mêmes immeubles, à la même date, sur les rôles d'évaluation. Voir infra, p. 42 
facture qui dépasse le cadre artisanal élémentaire. Il existe aussi une brasserie, mais nous ignorons son importance. Restent les chantiers de construction navale et les cours à bois, soit une vingtaine de gros employeurs ${ }^{22}$, qui embauchent sans doute la plupart des journaliers, ainsi que certains hommes de métier, tels que calfats, arrimeurs, «cullers» et charpentiers. Le secteur le mieux représenté est celui du bâtiment qui regroupe 96 menuisiers, 84 charpentiers, 44 maçons ainsi que des peintres, couvreurs, etc. Il compte plusieurs entrepreneurs qui conduisent des chantiers par toute la ville et composent l'élite du faubourg ${ }^{23}$.

Nous trouvons de véritables dynasties familiales dans chacun des principaux métiers et, dans bien des cas, nous pourrions remonter jusqu'à un grand-père recensé comme artisan à Québec en 1744, refoulé vers Saint-Roch après 1750 , à l'origine de toute une lignée professionnelle. L'essor démographique du faubourg doit énormément à l'excédent de population que la ville y déverse régulièrement, beaucoup aussi à la croissance naturelle de ses habitants et peut-être moins aux apports de la campagne, mais, cn l'âtsence de source sûre, cela demeure une impression ${ }^{24}$. Les habitants d'origine britannique sont relativement peu nombreux, moins de $10 \%$ des citoyens cotisés en 1821 , et d'après les recensements paroissiaux de 1795 et de 1805 la proportion de britanniques dans l'ensemble de la population est de $5 \%$ à Saint-Roch par rapport à environ $25 \%$ dans la ville ${ }^{25}$. Ceux qui descendent dans le faubourg, mis à part les quelques grands marchands-entrepreneurs, se concentrent dans les catégories journaliers et commerçants. Privilégiés pour l'obtention de permis, ils exploitent presque tous les cabarets et les auberges et, immigrants de fraîche date, souvent en transit vers le Haut-Canada ou les Etats-Unis, ils sont en majorité locataires.

22 Dominés par John Goudie. Voir aussi les entreprises de Thomas Lee, John Campbell, de Caldwell, Finch, etc.

23 D'après l'inventaire des marchés de construction dans l'ensemble de la ville, Bastien et coll., op. cit., passim. Lors de la création d'un régime municipal en 1833, ce sont deux charpentiers qui viennent représenter Saint-Roch à l'Hôtel de Ville. Drolet, op. cit., vol. III, 15.

24 Avant l'ouverture des registres paroissiaux de Saint-Roch, ses habitants relèvent de la paroisse Notre-Dame, qui englobe toute la ville et les faubourgs. Or les actes ne mentionnant jamais le quartier du domicile, il est impossible d'isoler la population du faubourg pour étudier sa formation.

25 John Hare, «La population de la ville de Québec 1795-1805», dans Histoire sociale (mai 1974): 27 et 38. 
Ce tableau rapide et bien incomplet n'avait pour but que de cerner la base sur laquelle repose le système de propriété qui est l'objet de cette étude: une masse de petits producteurs, propriétaires de leur logement et de leurs outils et qui, pour cette raison, forment un terrain idéal pour l'exercice de privilèges qui permettent de capter indirectement une bonne part du surproduit de leur travail.

\section{II - LE SYSTÈME DE PROPRIÉTÉ}

\section{L'introduction des rentes à bail d'héritage}

Henri Hiché, le premier lotisseur de Saint-Roch, vend ses terrains $3 \mathrm{~s} 4 \mathrm{~d}$, puis $4 \mathrm{~s}$ le pied carré à partir de 1751 , ce qui représente 5001 . pour un emplacement de 2500 pieds carrés, une somme considérable, supérieure à ce qu'un ouvrier peut gagner en deux ans. Comme celui-ci doit faire des déboursés immédiats pour la construction de sa maison, les aliénations ne peuvent que prendre la forme d'un crédit à long terme. Nous avons donc affaire à un double contrat, d'abord une vente, suivie de la création d'une rente au denier vingt (5\%) en faveur du vendeur. Mais c'est ici que l'opération se complique, car Hiché impose à son débiteur deux types de rente: soit une rente constituée pour les quatre-cinquièmes du principal et une rente foncière à bail d'héritage pour le reste. Voyons les choses de plus près en utilisant un exemple concret:

La vente faite «...moyennant le prix et somme de 5001 . monnaie de France, que ledit acquéreur a promis rendre, bailler et payer audit sieur vendeur, de prix principal en sa maison [...] par chacun an, la rente de 251 ., de laquelle rente ledit acquéreur, ses hoirs et ayant cause, pourront en amortir jusqu'à la somme de 4001 . toutes fois et quandes ils le jugeront à propos, en un seul paiement et non autrement, avec les arrérages qui seront dus alors. Et quant à la somme de 51 . restante de rente pour les autres 1001 . de principal, ledit acquéreur a promis et s'est obligé les bailler et payer audit sieur vendeur à titre de rente foncière de bail d'héritage non rachetable... ${ }^{26}$

La rente constituée de 201 . au principal de 400 1. est la forme de prêt à long terme communément pratiquée en France dès les débuts de l'ère moderne et qui gagne rapidement du terrain au

26 Actes de concession par $\mathrm{H}$. Hiché à André Chandonette et à Nicolas Godin, 18 mars 1751, Lanouillier, notaire: ANQ. 
Canada tout au long du XVIIIe siècle. La rente est dite perpétuelle, en ce sens que le créancier ne peut jamais exiger le remboursement. Elle est cependant rachetable au gré du débiteur. Les conditions de rachat sont stipulées dans le contrat, soit, dans le cas présent, en un seul paiement, condition la plus défavorable à l'extinction de la dette. La rente constituée est une dette personnelle de celui qui la crée ou de ses héritiers, mais elle est aussi garantie par une hypothèque spéciale sur le terrain vendu et une hypothèque générale sur tous les biens du débiteur. C'est son caractère "perpétuel» qui la distingue du prêt à intérêt pur et simple et contribue à la rendre donc licite, selon les canons de l'Église sur l'usure ${ }^{27}$.

La seconde rente de 51 . exigée par Hiché pour les 1001 . restantes est plus inattendue, car elle représente une charge réelle sur la propriété. Comme son nom l'indique, le bail d'héritage tient d'assez près au contrat de louage, puisque le bailleur, tout en cédant le terrain, retient des droits sur celui-ci et peut imposer pour la sûreté de sa rente toute une série d'obligations. Enfin cette rente esi non rachetabie, ce qui en fait une opération à fonds perdus, piutôt que de crédit, et accentue son caractère archaïque ${ }^{28}$. À peu près immobile depuis le Moyen âge, cette forme de transmission incomplète du droit de propriété n'est utilisée en principe en France que pour des cessions de biens ruraux, les ordonnances royales de 1441 et de 1553 ayant interdit la création de rentes non rachetables sur les immeubles parisiens d'abord et ceux des autres villes du royaume ensuite. Il y a cependant une exception en faveur des rentes «créées les premières après le cens» et n'excédant pas le tiers de la valeur de l'immeuble, sur laquelle Hiché s'appuie peut-être pour justifier son opération. Justification fragile toutefois, car la jurisprudence ne semble retenir l'exception que pour les rentes existantes, avant les ordonnances, et celles créées au profit des seigneurscensiers $^{29}$. Or ici la création est postérieure et Hiché n'est qu'un censitaire sur cette terre relevant du domaine royal.

Mais légales ou non, à quoi riment ces subtilités? Pourquoi un propriétaire, qui fait montre d'autre part d'un bon sens des affaires, va-t-il déterrer une forme d'aliénation, qui, dans ce contexte

27 Philippe-Antoine Merlin dit Merlin de Douai, Répertoire universel et raisonné de jurisprudence, 5e éd. (Bruxelles, 1828-30), vol. 28: 198-351; Oeuvres de Pothier, publiées par M. Bugnet (Paris, 1847), vol. 3: 436-511; B. Schnapper, Les rentes au XVIe siècle (Paris, 1958).

28 Pothier, op. cit., vol. 4: 171-240 et Schnapper, op. cit., passim.

29 Pothier, op. cit., vol. 4: 180-181; et Schnapper, op. cit., 231-232 et notes 19 et 20. 
urbain et colonial du XVIIIe siècle, a l'allure d'une curiosité juridique? La réponse est inscrite dans la liste des charges imposées à l'acheteur: porter sa rente chaque année à la Saint-Michel; faire clore par moitié avec les voisins et à ses seuls dépens si le terrain touche à celui du vendeur; entretenir la rue au-devant de l'emplacement; bâtir une maison et tenir feu et lieu dans un an et un jour du contrat à peine de nullité de ce dernier; fournir à ses frais une grosse du contrat. Enfin, le rentier se réserve un "droit de retrait» en cas de vente de l'immeuble ${ }^{30}$. Il est normal qu'un lotisseur pose certaines conditions portant sur la forme et la destination des immeubles, la qualité des matériaux, etc., dans le but de contrôler l'occupation du quartier et maintenir ainsi la valeur des terrains non encore vendus ${ }^{31}$. Mais ces préoccupations d'ordre spéculatif n'entrent pas en ligne de compte à Saint-Roch. Ici le lotisseur ne se soucie pas de l'aspect du quartier, mais cherche simplement à assurer la sécurité de ses rentes et à faire porter sur les acheteurs l'aménagement du faubourg. Pour ce faire, il calque ses contrats sur les baux à cens en usage dans la colonie, conçus pour atteindre les mêmes objectifs.

Assimilées à juste titre aux privilèges féodaux, les rentes foncières non rachetables, qui donnent au créancier les mêmes droits qu'un seigneur sur son fief, furent supprimées en France dans la nuit du 4 août $1789^{32}$. Au Canada, accolées au mouvement d'urbanisation, elles survivent jusqu'en $1859^{33}$.

Hiché meurt avant la fin de la guerre et ses héritiers cherchent à liquider leurs biens avant d'émigrer en France ${ }^{34}$. Mais pour trouver un acheteur, il faut essayer de mettre un peu d'ordre dans le

30 Voir infra, sous-chapitre 5, pour une analyse de ces droits.

31 C'est le cas, par exemple, des baux emphytéotiques fréquemment utilisés à cette époque dans les villes européennes et américaines par lesquels le preneur s'engage à faire les grosses réparations ou à construire tel type de maison, là où seul le terrain est fourni. La valeur ainsi créée revient au propriétaire lorsqu'il récupère le fonds à la fin de ce bail à long terme. Voir Donald J. Olsen, Town Planning in London: the Eighteenth and Nineteenth Centuries (Yale U.P., 1964). Pour d'autres formes de lotissement, voir Sam B. Warner, jr., Streetcar Suburbs: the Process of Growth in Boston 1870-1900 (New York, 1973), chap. 6; et aussi J.-P. Bardet, «La maison rouennaise aux XVIIe et XVIIIe siècles. Economie et comportement", 363-367, dans Le bâtiment. Enquête d'histoire économique, XIV-XIXe siècles, tome I, Maisons rurales et urbaines dans la France traditionnelle (Mouton, 1971).

32 Merlin de Douai, op. cit., vol. 28: 220.

33 Loi 22 Victoria, ch. 49, s. 1 (1859); B. A. Testard de Montigny, Histoire du droit canadien (Montréal, 1869), 317.

34 Hiché meurt à Québec le 15 juillet 1758. Il laisse trois enfants: un fils marié qui meurt en France en 1762, Madeleine épouse d'Ignace Perthuis, négociant et une autre fille religieuse à l'Hôpital général, seul membre de la famille à rester au Canada. P.-G. Roy, op. cit. 
faubourg ruiné et déserté ${ }^{35}$. La famille obtient du gouverneur anglais une ordonnance, publiée aux quatre coins du quartier, sommant les habitants, qui sous prétexte que leurs maisons ont été détruites ont négligé d'acquitter les rentes, de se présenter avec leurs titres pour payer les arrérages, à peine de se voir déchoir de leur propriété ${ }^{36}$. Douze emplacements sont vendus par les Hiché entre 1762 et 1764 , selon les mêmes modalités, mais à raison de $5 \mathrm{~s}$ et $6 \mathrm{~s}$ le pied carré ${ }^{37}$. Un acheteur se présente enfin. L'affaire est mauvaise mais le temps presse, le délai que les traités accordent aux partants pour réaliser leurs propriétés tirant à sa fin. Tout le pays est à vendre et les acheteurs mènent le jeu. C'est ainsi que William Grant, ancien munitionnaire de l'armée anglaise, devient propriétaire de Saint-Roch, ce qui comprend 130 rentes foncières et constituées, 72 arpents [ 26 ha] non lotis avec la résidence et ses dépendances ${ }^{38}$.

\section{La phase seigneuriale}

Grant s'installe à la Maison Blanche, exploite trois moulins qui par la rivière Saint-Charles reçoivent des grains de toute la colonie $^{39}$ et poursuit à rythme ralenti le lotissement de la métairie, à raison de $6 \mathrm{~s}$ le pied carré. Les contrats de vente de cette époque prennent un nouveau tour, fort surprenant. Voilà Saint-Roch promu au rang de "fief», la Maison Blanche devenue «manoir", d'où le «Seigneur» baille ses terrains à titre de «cens et rentes foncières et seigneuriales perpétuelles et non rachetables ${ }^{40}$. Or per-

35 Voir entre autres le journal de Jean-Claude Panet qui décrit les bombardements de Saint-Roch, Journal du siège de Québec en 1759 (Montréal, 1866).

36 Ordonnance de James Murray, 13 septembre 1763, déposée dans le greffe de J.-C. Panet, notaire, ANQ.

37 Lemaître de La Morille, notaire, juillet 1762 - juin 1764, ANQ.

38 Contrat de vente du 29 septembre 1764 ratifié après diverses procédures le 24 juin 1766, notaire J.-C. Panet, ANQ. Il est difficile de connaître le prix de vente car, d'une part, il comprend aussi la propriété des Perthuis dans la ville et, d'autre part, il est acquitté en «billets d'ordonnance», monnaie dépréciée qui inonde alors la colonie, et deux rentes viagères. En 1810, le receveur du Domaine, voulant calculer les lods et ventes qui lui sont dus sur cette transaction, ramène toute la somme à un douzième de sa valeur nominale. Les Hiché auraient donc touché environ 230001 ., à supposer qu'ils aient réussi à convertir à temps ces billets, une somme dérisoire puisque, avant la guerre, la rente de Saint-Roch à elle seule rapportait 3500 1. Rapport de Jos. Planté, receveur, 21 février 1810: ANQ, QBC 17-1, pièce 78.

39 Deux moulins à vent et un à eau: plan non daté dans APC, RG1, L3L, fo 47666.

40 Voir les actes de concession passés par Grant dans les greffes de Louis Descheneaux (1781-1794) et Félix Têtu (1794-1805): ANQ. 
sonne n'ignore, surtout pas les notaires complices du nouveau propriétaire, que cette terre n'est qu'une roture dans la directe du roi. Il s'agit bel et bien d'une usurpation de titre que Grant, grâce à son prestige de conseiller législatif, réussit à faire sanctionner en 1781 par un acte de foi et hommage enregistré par un fonctionnaire complaisant ${ }^{41}$.

Il est possible que le titre flatte sa vanité, mais il y a plus. Cette mutation sémantique, loin d'être anodine, permet d'abord l'appropriation des droits de cens et surtout de lods et ventes, aux dépens de la Couronne, et assure au propriétaire une meilleure emprise sur les habitants du faubourg. De nouvelles obligations viennent s'ajouter aux anciennes, ainsi, celle de rebâtir la maison dans les trois ans suivant un incendie ou autre destruction, à peine de «réunion au domaine». Témoin des problèmes des Hiché après la guerre, Grant s'arme pour affronter les catastrophes. Les contrats précisent en outre que le seigneur peut reprendre le terrain, sans formalités de justice, dans le cas où l'acheteur resterait trois ans sans tenir feu et lieu et laisserait ses rentes tomber en souffrance. Enfin, il est interdit de diviser les cens et rentes à la suite d'une vente ou d'un partage sans le consentement du vendeur. Dernière particularité, les conditions de rachat de la rente constituée, qui subsiste pour la majeure partie du principal, n'apparaissent nulle part dans ces contrats volontairement ambigus, qui mélangent habilement la dette personnelle et les charges réelles ${ }^{43}$.

Les titres de Saint-Roch ne furent pas contestés avant la fin du siècle. En 1798, une poursuite pour non paiement des lods et ventes intentée contre un soi-disant censitaire de Grant fut portée à l'attention du procureur général, John Sewell ${ }^{44}$. Ménagements ou simples lenteurs administratives, ce n'est que sept ans plus tard, s'appuyant sur l'opinion de ce dernier, que la Couronne entreprit les poursuites pour récupérer les droits seigneuriaux que Grant avait

41 La réussite de William Grant est rapide. En 1770, il épouse la veuve du baron de Longueuil et est nommé receveur général de la colonie, puis conseiller législatif en 1774. Il achète, d'autre part, plusieurs seigneuries rurales. Voir P.-G. Roy, Inventaire des concessions en fief et seigneurie, passim. Roy a d'ailleurs noté l'usurpation de titres à Saint-Roch: vol. I: 16-17:

43 Grant comme ses successeurs utilise des formules imprimées spécialement pour les ventes d'emplacements du faubourg. Voir à titre d'exemple, le contrat du 31 juillet 1807 , notaire Jean Bélanger, ANQ.

44 Poursuite contre Augustin Borneau, navigateur domicilié à Saint-Roch, Cour du Banc du Roi, 30 janvier 1978: ANQ, Archives privées, série Seigneuries, Saint-Roch. 
illégalement perçus dans le faubourg ${ }^{45}$. Sewell s'était aussi penché sur le cas des rentes foncières non rachetables pour conclure que, ayant été interdites par les rois de France au XVIe siècle, il serait souhaitable de faire disparaître cet abus au Canada.

$\mathrm{Au}$ cours de ces longues procédures, ni les magistrats ni les fonctionnaires n'avaient soulevé la question des retombées de ces pratiques irrégulières sur les habitants de Saint-Roch. Sitôt le jugement signifié, le notaire se contente de biffer les mots «fief, cens et seigneurial» sur les anciennes formules et les aliénations suivantes portent encore une part de rente foncière non rachetable, comme au temps de Hiché ${ }^{46}$. Toutefois, après le morcellement de la propriété du faubourg en 1810, un nouveau lotisseur, John Mure, va devancer la législation et éliminer la rente foncière de ses contrats. Ceux qui achètent leur emplacement de ce propriétaire ne créent plus qu'une seule rente constituée, donc rachetable, pour le plein montant de l'acquisition. En principe, la plupart des servitudes imposées naguère aux débiteurs, qui découlaient de la nature du bail d'héritage, devraient alors disparaître. Mais il n'en est rien. Les rentes constituées en faveur du nouveau créancier restent assorties des mêmes charges et privilèges que par le passé ${ }^{47}$.

\section{Les derniers lotissements, 1810-1825}

En 1810 Saint-Roch est vendu à la poursuite des créanciers de la succession Grant. Les quelque 475 rentes créées avant cette date sont offertes aux enchères par lots variant entre 100 et 150 terrains contigus. John Richardson, négociant et conseiller législatif, déjà présent dans Saint-Roch à titre de curateur à la succession, conserve tout le terrain encore vacant au nord du faubourg, prolongé par la rive de la Saint-Charles que le gouvernement vient de lui concéder ${ }^{48}$. Il distribue rapidement les terrains en bordure de la rivière aux constructeurs de navires et aux exportateurs de bois et vend des emplacements domiciliaires sur les rues Queen et Prince

45 Voir l'opinion de Sewell et la correspondance de l'inspecteur du Domaine, 1805 1810 dans ANQ, QBC, 17-1, pièces $27,38,39,75$ et 78 . Grant meurt en 1805 et ce sont ses exécuteurs qui sont condamnés à rembourser £9 729. 11sh 8d [233 510 1.] par les sentences des 20 juin et 29 juillet 1809 . Les bases de cette évaluation ne sont pas fournies. Le curateur fit opposition et nous ignorons comment l'affaire se termina. Voir ANQ, Archives privées, série Seigneuries, Saint-Roch et l'acte du 29 juillet 1809 dans le greffe du notaire Bélanger.

46 Voir les ventes passées devant le notaire Bélanger après le 5 août 1809 .

47 Par exemple, vente d'un emplacement par John Mure à A. Bornais, charpentier, 20 juillet 1810: J. Bélanger, notaire, ANQ.

48 Voir supra, note 9. 
Edward, juste derrière. La dernière section encore non lotie, dans l'angle sud-ouest du faubourg, est adjugée à John Mure, un autre négociant et constructeur de navires, député de la Basse-Ville et, plus tard, conseiller législatif. Mure paie $£ 2095$ [50 280 1.] pour 799270 pieds carrés ${ }^{49}$ et procède immédiatement à des ventes massives d'emplacements: 80 en 1810-1811 et le reste, soit 85 , entre 1816 et $1825^{50}$. Ainsi achève-t-on de disposer de l'ancienne terre de la Pointe-aux-Lièvres. Le tableau 3 et la carte qui suivent résument de manière approximative les principales étapes de ce lotissement étalé sur un siècle.

TABLEAU 3

LOTISSEMENT DU FAUBOURG SAINT-ROCH, 1731-1825*

\begin{tabular}{|l|l|c|cc|}
\hline Période & Lotisseur & Nombre d'empla- & \multicolumn{2}{|c|}{ Superficie } \\
\hline $1731-1765$ & cements vendus & Arpents & $\%$ \\
$1765-1805$ & W. Hiché & 141 & 12 & 14 \\
$1805-1810$ & Wuccession Grant & $(180)$ & $(14)$ & $(16)$ \\
$1810-1825$ & J. Richardson & $(150)$ & $(23)$ & 14 \\
$1810-1825$ & J. Mure & 165 & 25 & 29 \\
TOTAL & & $(788)$ & 86 & 100 \\
\hline
\end{tabular}

* Reconstitution approximative. Les chiffres entre parenthèses correspondent aux deux périodes où nous n'avons pas pu faire un relevé complet des ventes, distribuées chez différents notaires. Notons aussi que Mure concède un très grand terrain pour l'église, ce qui affecte le rapport entre le nombre d'emplacements et la superficie.

\section{Le rendement de la rente}

Depuis le début du lotissement le prix de vente des emplacements poursuit une marche ascendante et il est intéressant d'observer que la dernière hausse, la plus importante, se situe au moment où les prix et les salaires commencent à fléchir (voir tableau 4). Le mouvement de baisse générale s'accentuant jusqu'au milieu du siè-

49 Contrat du 2 mai 1810, suite à l'adjudication du 14 avril, déposé chez Berthelot, notaire, ANQ. Le marchand Etienne-Claude Lagueux finance l'acquisition de Mure et retient comme garantie une hypothèque sur les rentes qui seront créées sur le terrain.

50 Avis du shérif de la vente aux enchères de 165 rentes constituées en faveur de l'Hon. John Mure (comprenant les noms des débit-rentiers, la rue, les dimensions des emplacements, le montant des rentes et la date de création): extrait de la Gazette de Québec, 17 août 1825, Papiers Ross, Archives du musée McCord, Montréal. 
ÉTAPES DU LOTISSEMENT DU FAUBOURG SAINT-ROCH, 1731-1825

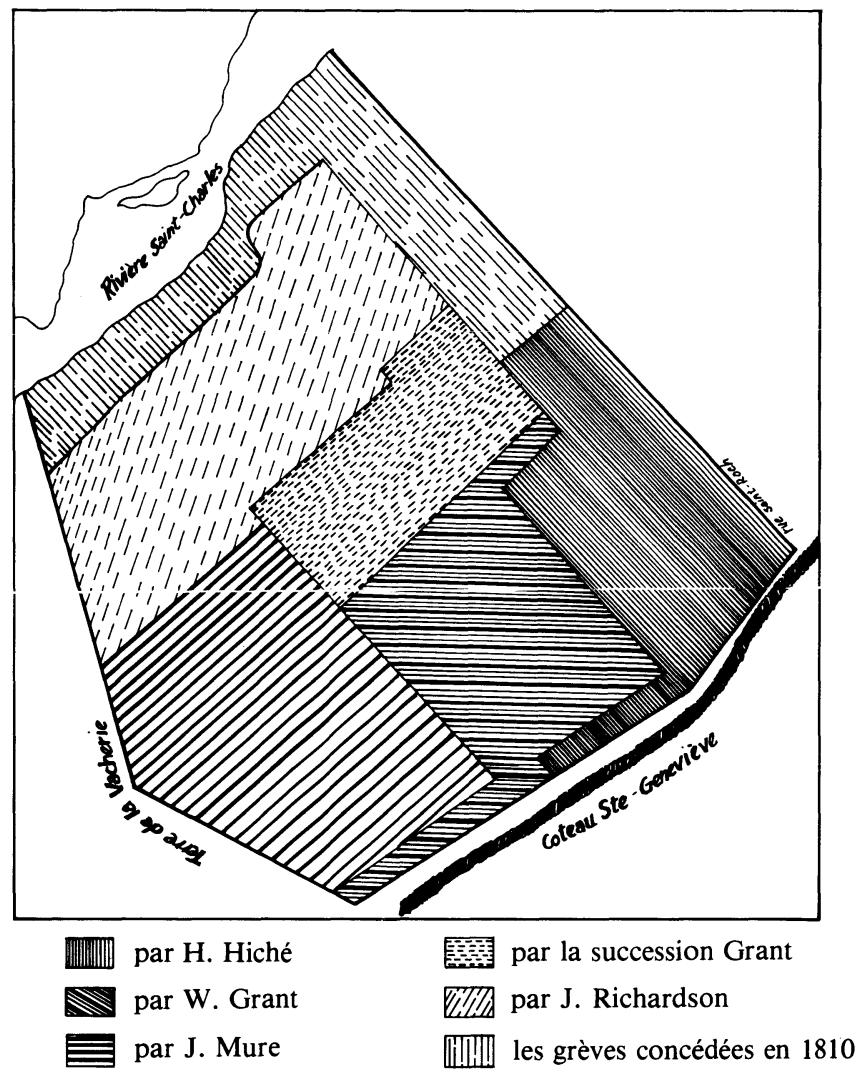

cle, la rente représente un poids de plus en plus lourd pour les débiteurs du faubourg et un placement privilégié pour les créditsrentiers.

En achetant ces 25 arpents dans Saint-Roch, John Mure fit une bonne affaire. Le terrain vacant lui revenait à $1 \mathrm{~s}$. $6 \mathrm{~d}$. le pied carré et il revendit rapidement des emplacements $8 \mathrm{~s}$. et $12 \mathrm{~s}$. le pied carré. Avec un tel écart, quelques années de rentes suffisaient pour rembourser sa mise de fonds. A sa mort, quinze ans plus tard, les 165 rentes créées en sa faveur, avec treize emplacements 
TABLEAU 4

ÉVOLUTION DU PRIX DE VENTE DES EMPLACEMENTS DE SAINT-ROCH, 1731-1825

\begin{tabular}{|c|c|c|}
\hline Périodes & $\begin{array}{c}\text { Prix moyen par pied carré } \\
\text { (monnaie de France) }\end{array}$ & $\begin{array}{c}\text { Charge annuelle pour 2 } 500 \\
\text { pieds carrés taux: } 5 \%\end{array}$ \\
\hline $1731-1750$ & $3 \mathrm{s.} 4 \mathrm{~d}$. & $211.8 \mathrm{~s}$. \\
$1751-1758$ & $4 \mathrm{~s}$. & 251. \\
$1762-1764$ & $5 \mathrm{~s}$. & $311.5 \mathrm{~s}$. \\
$1765-1810$ & $6 \mathrm{~s}$. & $371.1 \mathrm{~s}$. \\
$1810-1811$ & $8 \mathrm{~s}$. & 501. \\
$1816-1825$ & $12 \mathrm{~s}$. & 751. \\
\hline
\end{tabular}

Source: contrats de ventes chez les différents notaires cités au cours de cet article.

restants, furent vendus en bloc pour $£ 6000$ [144 000 1.], un profit net pour ses héritiers ${ }^{51}$.

Mais les transactions sur la rente couvrent autre chose qu'une simple spéculation foncière. En 1810, Joseph Roy, bourgeois de Québec, achète 142 rentes dans Saint-Roch pour £1810 [43 440 1.], alors que la valeur capitalisée de ces rentes, au taux de $5 \%$, est de $822721 .^{52}$. Pareillement, lorsque George Pozer acquiert en 1825 les 65 rentes de John Mure au revenu annuel de 130191 ., il paie $£ 5750$ ou $1380001 .{ }^{53}$. Ainsi la valeur capitalisée des rentes est évaluée sur le marché à la moitié du prix de vente des emplacements. Ce prix de vente n'est en fait une réalité que pour l'habitant de Saint-Roch, qui doit débourser la pleine valeur, soit, par exemple, 10001 . pour racheter la rente de 501 . qui grève son emplacement, alors que le prix courant de cette rente, partant du terrain, est de 500 1. Cette dissociation nous ramène au caractère spécifique de ce lotissement qui, à partir d'une situation de monopole, organise la vente des emplacements en marge du marché.

Le placement de ces rentiers produit donc un revenu de $10 \%$ et c'est ce deuxième taux de capitalisation qu'il faudrait expliquer,

51 Contrat du 4 janvier 1825, notaire Jean Bélanger, ANQ.

52 Contrat du 26 novembre 1810 , notaire J. Bélanger, ANQ.

53 Contrat du 4 janvier 1825 , ibid. Pozer paie $£ 5750$ pour les rentes et arrérages et $£ 250$ pour 13 emplacements non encore vendus. Cette vente est suivie d'un décret volontaire, en 1826 , procédure quasi routinière pour forcer les créanciers hypothécaires à se manifester, le cas échéant. 
par référence au taux d'intérêt moyen du capital investi dans les maisons de rapport de la ville, car les deux sont évidemment liés. Le taux élevé de la rente de Saint-Roch laisse entrevoir des revenus locatifs bruts supérieurs à $10 \%$ puisque, normalement, le capital immobilisé dans la rente est de moindre rapport que celui placé dans des immeubles aisément réalisables. Il n'existe encore aucune étude sur les loyers québécois pour asseoir une telle comparaison. L'analyse des actifs de Pozer nous fournit cependant un indice. Lorsque celui-ci achète des rentes dans le faubourg, il est déjà propriétaire de 19 maisons à la Haute-Ville et à la Basse-Ville et nous connaissons la valeur engagée, entre 1793 et 1809, dans dix d'entre elles $^{54}$, ainsi que leur valeur locative en 1821 , laquelle représente $16 \%$ du prix d'achat. Compte tenu de l'absence de synchronisme entre les deux termes, il ne s'agit évidemment pas du revenu locatif réel $^{55}$, mais ne peut-on pas lire dans ce rapport démesuré la trace des bouleversements qui auraient affecté le marché immobilier au début du siècle? Imaginons d'abord un marché faible qui n'attire guère les capitauxix, quìi sônt hâppés pâl une conjoncture commerciale houleuse mais dans l'ensemble favorable et par les placements ruraux, et où le prix de la propriété urbaine stagne. Puis, avec la fin de la guerre et l'arrivée à Québec de milliers d'immigrants ${ }^{56}$, la demande subite de logements fait grimper les loyers. Ce mouvement ne peut alors que stimuler les investissements dans les immeubles anciens comme dans la construction, d'autant plus que la récession dans le secteur des importations invite au repliement, et aussitôt les loyers commencent à redescendre vers un niveau plus normal. Si le scénario est juste, il expliquerait en même temps le taux élevé des rentes, accordé à celui des loyers, et l'attrait que représente ce revenu fixe au moment où le mouvement bifurque vers la baisse.

Même élevés, les loyers ne sont jamais de tout repos. Ils ne sont garantis que par la personne du locataire, sa réputation et la solidité de son commerce. Le propriétaire doit souffrir les retards et, lorsqu'il se résout à intenter une action, les meubles ont été vendus et il n'y a plus rien à saisir. Les rentrées des rentes sont égale-

54 Quatre achats ont été relevés dans les actes notariés et six dans les ventes publiques par le shérif: archives du ministère de la Justice, à Québec. Ce dernier mode d'acquisition est sans doute plus avantageux que la vente de gré à gré.

55 Voir J.-P. Bardet, op. cit., 368-369.

56 Soit environ 10000 par année au port de Québec entre 1816 et 1826 . Voir F. Ouellet, Histoire économique et sociale du Québec, 1760-1850 (Montréal, 1966), graphique, 601 . 
ment irrégulières mais, même si les mauvaises années voient s'étirer les délais, la dette demeure garantie par la propriété bâtie, qui excède de loin la mise de fonds du rentier, et par tout ce corset de droits qui empêchent la prescription des arrérages.

\section{La conservation du patrimoine}

La principale différence entre ces deux types de propriété ne réside toutefois pas dans leurs rendements respectifs, mais plutôt dans la nature même de ces placements et nous faussons sans doute la réalité en essayant d'appréhender la rente de Saint-Roch avec des concepts économiques qui lui sont étrangers. Elle appartient à un monde de rigidité et de lenteurs, qui puise sa rationalité, non pas tant dans le profit, que dans la conservation du patrimoine. Afin d'éclairer cet aspect, nous avons étudié de près la gestion de George Pozer entre 1825 et 1846. C'est en examinant la façon dont le rentier tire parti de ses droits que nous pouvons mieux comprendre l'utilité des réserves et privilèges inscrits dans les contrats et saisir l'écart qui sépare ce placement des autres opérations foncières articulées au mode de production capitaliste.

Pozer commence par retenir les services du notaire Jean Bélanger, bien versé dans les affaires du faubourg ${ }^{57}$. Celui-ci peut recevoir les rentes et poursuivre les débiteurs défaillants. Il doit, dans tous les cas de mutation des emplacements, requérir «titre nouvel» des acheteurs, héritiers ou ayants cause. Il assistera aux ventes publiques des propriétés du faubourg, pourra couvrir l'enchère et même la remporter «pour éviter toutes pertes»" ${ }^{58}$. En dépit de ce mandat, Pozer s'occupe personnellement de ses affaires car, contrairement à ses prédécesseurs, négociants et hommes publics, le nouveau propriétaire de Saint-Roch est retiré du commerce et peut consacrer tout son temps à l'administration de sa fortune ${ }^{59}$. Mais en définissant la nature des interventions, cette procuration nous fournit un fil directeur.

57 Bélanger a travaillé pour John Richardson au règlement de la succession de W. Grant et aussi pour John Mure. C'est lui également qui s'occupera du lotissement de la terre de la Vacherie, dans le prolongement de Saint-Roch, après 1825.

58 Procuration du 5 février 1825: Jacques Voyer, notaire. ANO.

59 D'origine allemande, établi à Québec en 1785, Pozer fut marchand-épicier et prêteur. Les profits furent investis dans de nombreuses propriétés urbaines et rurales qui représentent à sa mort, en 1848 , une fortune considérable. Nous avons préparé un article sur le personnage qui paraîtra dans le vol. VII du Dictionnaire Biographique du Canada. C'est d'ailleurs à partir de cette recherche sur Pozer que nous nous sommes intéressée aux rentes de Saint-Roch et avons remonté la filière jusqu'à leur origine. 
Quiconque a fréquenté les greffes des notaires canadiens du XIXe siècle a certainement été frappé par la grande quantité d'actes intitulés «titres nouvels». Il s'agit d'un acte par lequel le nouveau propriétaire d'un immeuble reconnaît être débiteur de la rente qui grève cet immeuble et accepte toutes les charges du contrat primitif. Il est souvent complété par une obligation à demande pour les arrérages ${ }^{60}$. C'est l'absence de régime officiel de publicité des hypothèques ${ }^{61}$ qui rend cette précaution nécessaire, car les droits que le crédit-rentier possède sur le débiteur sont en péril à chaque mutation de propriétaire. S'il s'agit d'une succession, la rente risque d'être fractionnée entre plusieurs héritiers, ce qui compromet la perception. On exige alors un «titre nouvel» pour assurer l'indivisibilité de la rente ${ }^{62}$. Le danger est plus grand quand la propriété est vendue puisque, si le transfert de la dette n'est pas stipulé dans le contrat de vente, le rentier n'a aucun recours contre le tiers détenteur pour les arrérages et celui-ci peut choisir de déguerpir lorsque sommé de payer la rente ${ }^{63}$. Le recours que le rentier conserve contre le vendeur est peu utile, comptc teniu de l'insolvabilité probable de celui qui vient de se départir de son bien. Le notaire doit donc surveiller les mutations et exiger une reconnaissance de titre de tous les nouveaux propriétaires. S'ils refusent, le rentier brandit son "droit de réunion» ou droit de reprendre l'emplacement abandonné par le propriétaire primitif, dans une action «en passation de titre nouvel» et le jugement tient lieu de nouveau titre si le défendeur persiste dans son entêtement ${ }^{64}$.

Plus que les divisions et les aliénations de propriété, c'est la vente en justice qui peut compromettre la sécurité de la rente. Lorsqu'un habitant de Saint-Roch est poursuivi par des créanciers et que sa propriété est mise aux enchères, la procédure a pour effet d'amortir immédiatement la rente ${ }^{65}$. L'adjudicataire paie les créanciers suivant l'ordre établi par la sentence, une fois réglées les

60 Voir par exemple les «titres nouvels» passés par John Child, notaire, tout au long de l'année 1843, ou ceux des 26 janvier 1844 et 14 février 1846: ANQ.

61 Ce n'est qu'en 1841, par l'ordonnance du Conseil Spécial, 4 Vict. ch. 30 , que ce régime officiel est instauré dans l'ensemble du Bas-Canada.

62 Dans le cas très fréquent de propriété indivise, chaque héritier reconnaît devoir le montant entier de la rente.

63 Il est évident que l'existence de la rente ne peut pas être cachée si le contrat est rédigé par un notaire du faubourg mais un vendeur pourrait tenter d'échapper à cette surveillance pour tromper l'acheteur.

64 Action intentée par G. Pozer contre Judith Chartier, veuve Lacoste et ses enfants, Cour du Banc du Roi, 22 février 1844: Papiers Ross, Musée McCord.

65 On dit que la criée des immeubles «purge les hypothèques». 
créances privilégiées telles que douaires, gages d'ouvriers, droits seigneuriaux au Domaine royal. Quelles que soient les circonstances, le principal de la rente risque d'être évalué au prix courant plutôt qu'à sa valeur nominale originelle. Si l'enchère ne monte pas, c'est une somme plus médiocre encore qui sera attribuée au rentier et le décret lui a fait perdre tout recours ultérieur contre son débiteur.

Nous voyons donc pourquoi le rentier n'intente à peu près jamais de saisies, fait preuve d'une telle tolérance envers les débiteurs. Plutôt attendre des temps plus favorables en conservant tous ses droits que de jeter ceux-ci dans une mêlée générale de créanciers, d'où il sortirait avec un patrimoine amoindri. Impuissant lorsque l'action en saisie est intentée par un tiers, il est alors forcé de se porter lui-même adjudicataire pour éviter le pire. Rien ne s'oppose ensuite à ce qu'il rende la propriété au saisi et, en fait, c'est souvent le meilleur arrangement. Jouant sur la reconnaissance de celui qui s'estime heureux d'avoir évité l'expulsion, il lui revend son terrain et sa maison, avec création d'une nouvelle constitution de rente et lui fait signer en même temps une obligation pour les frais de cour et les arrérages du passé, qui, par ce détour, refont surface ${ }^{66}$.

Dans une série incomplète de ventes publiques, nous avons relevé huit cas où Pozer remporte l'adjudication d'un emplacement de Saint-Roch sur lequel il détient une rente et dans tous, sauf un, la poursuite a été intentée par un tiers ${ }^{67}$. Les droits inscrits dans le contrat de vente donnent d'ailleurs au rentier des moyens d'intimidation pour reprendre la propriété d'un débiteur obéré sans formalités judiciaires, ce qui est entériné par un acte «d'abandon» ou de «délaissement». Quelle que soit la procédure, la reprise peut évidemment être une source de profit ${ }^{68}$.

66 Contrat de vente du 4 avril 1846 à Thomas Darbyson, débardeur: John Childs, notaire, ANQ; ou encore, l'adjudication de la propriété de François Lemelin, chartier, du 30 mars 1839, laquelle est revendue par Pozer au fils Lemelin, le 17 août suivant: ANQ, QBC, série 28 , vol. 46. sim.

67 Ces ventes sont échelonnées entre 1831 et 1837: ANQ, QBC, série 28, vol. 46, pas-

68 Pierre Lambert délaisse sa propriété à George Pozer «en sa qualité de seigneur foncier de cette partie de Saint-Roch"), moyennant 1584 1. et se décharge ainsi d'une rente constituée de 370 1. Pozer la revend à Gervais Emond qui crée une nouvelle rente de 432 1., augmentation justifiée par la présence d'une maison sur le terrain: vente du 31 octobre 1846, Jacques Voyer, notaire, ANQ. 
Mais Pozer n'est pas à l'affût de ces sortes de transactions car, durant les vingt et une années que nous avons observées, seulement 32 nouvelles rentes sont créées, pour une somme globale de 2640 1. L'assise des revenus demeure remarquablement stable, comme le montre la comparaison rue par rue entre les rentes achetées en 1825 et celles qu'il donne à son petit-fils en 1846 . Les variations somme toute mineures découlent de quelques saisies ou abandons suivis de reventes, de réajustements à la suite de la subdivision ou du remembrement des terrains et de rares amortissements volontaires ${ }^{69}$. Mais il est clair que les habitants du faubourg n'ont pas souvent les moyens de racheter la rente et d'ailleurs nous savons que Pozer fait tout pour les en dissuader ${ }^{70}$.

TABLEAU 5

ÉTAT DES RENTES DE GEORGE POZER DANS SAINT-ROCH, EN 1825 ET 1846

\begin{tabular}{|l|c|c|c|c|}
\hline \multirow{2}{*}{ Rues } & \multicolumn{2}{|c|}{$1 \overline{8} 25$} & \multicolumn{2}{c|}{1846} \\
\cline { 2 - 5 } & $\begin{array}{c}\text { Nombre de } \\
\text { rentes }\end{array}$ & Revenu & $\begin{array}{c}\text { Nombre de } \\
\text { rentes }\end{array}$ & Revenu \\
\hline Fleury & 13 & 1086 & 19 & 1098 \\
Sainte-Hélène & 12 & 810 & 14 & 948 \\
Sainte-Marguerite & 29 & 1954 & 28 & 1948 \\
Des Fossés & 29 & 2162 & 31 & 2495 \\
Saint-Joseph & 19 & 2242 & 19 & 2384 \\
Saint-François & 18 & 1867 & 14 & 1171 \\
King & 23 & 1324 & 26 & 1498 \\
Richardson & 15 & 777 & 18 & 804 \\
Anne & 5 & 351 & 5 & 351 \\
Saint-Vallier & 2 & 446 & 1 & 326 \\
TOTAL & 165 & 13019 & 175 & 13023 \\
\hline
\end{tabular}

Source: Gazette de Québec, 17 août 1825, Papier Ross, Archives du Musée McCord; acte de donation de G. Pozer à G. Alford, 5 octobre 1846, notaire John Childs, ANQ.

69 Les rentes qui grevaient des terrains concédés par John Mure à l'évêque de Québec, pour servir à la nouvelle paroisse, furent rachetées du temps de Pozer. D'autres rachats de rentes ont pu nous échapper, mais il est certain qu'ils sont exceptionnels.

70 Par exemple, Jane Scott, confiseuse, rapporte que Pozer fut fort mécontent lorsqu'elle se présenta chez lui avec le principal d'une rente de 4201 . créée dix ans auparavant: témoignage du 9 février 1854, procès Southern vs Alford, Cour supérieure, Papiers Ross, Archives du Musée McCord. 
Le droit de retrait, qui figure dans tous les titres du faubourg, accorde au rentier le privilège de se substituer à l'acheteur d'un emplacement, dans les 40 jours suivant la vente, en remboursant à ce dernier le prix et loyaux coûts ${ }^{71}$. En somme il offre la possibilité d'acheter à bon compte, en faisant fuir les acheteurs, et de revendre avec profit. Or, nous n'avons pas trouvé d'exemples de ces achats qui, le cas échéant, se traduiraient par la création de nouvelles rentes au moment de la revente. Mais à voir l'insistance avec laquelle Pozer presse son notaire d'insérer ce droit dans les contrats, il est impossible de le tenir pour lettre morte. Le rentier peut en effet intervenir dans une vente sans se porter acquéreur. La menace du retrait est sans doute assez efficace pour éviter d'avoir à retraire et ainsi, grâce à cette arme de chantage, le propriétaire éminent peut bloquer une transaction, évincer tel acheteur quand il le juge utile pour ses intérêts, tractations qui ne laissent évidemment aucune trace.

Nous ne croyons pas que cet exposé des usages juridiques et procéduriers soit superflu. Seule en effet l'étude technique des pratiques coutumières permet de mettre en lumière le caractère spécifique de cette forme de propriété. Elle montre que le rentier ne cherche pas tant à faire fructifier un capital qu'à le conserver et, pour ce faire, tous les moyens qu'il déploie visent à reproduire les rapports sociaux, à resserrer un contrôle de la propriété qui passe par le contrôle des personnes, confusion essentiellement féodale que la Coutume prolonge bien avant dans les temps modernes ${ }^{72}$.

$$
* * *
$$

La valeur et la sécurité des rentes de Saint-Roch reposent sur la capacité de l'artisan ou du petit commerçant à produire au-delà de ce qui est nécessaire pour renouveler ses conditions de vie et de travail et, en même temps, sur la faiblesse et l'irrégularité de ce surplus, qui lui interdisent d'amortir sa dette. Ces petites gens n'achètent pas vraiment leur emplacement, mais plutôt un droit d'usage sur celui-ci, soumis à l'obligation d'y construire une maison qui servira de support à la rente.

71. Selon la Coutume de Paris, qui a cours au Canada, le retrait serait réservé aux parents lignagers. Par un abus qui remonte au XVIIe siècle, les seigneurs canadiens ont introduit le retrait dit féodal dans les baux à cens. L'usage qui étend ce droit aux simples rentiers est donc doublement abusif.

72 Sur cet aspect fondamental du féodalisme, lire Alain Guerreau, Le féodalisme, un horizon théorique (Paris, 1980), 177-184. 
Cette rente ne représente pas une charge négligeable. La majorité des propriétaires du faubourg doivent verser annuellement entre 37 et 751 ., selon la date du titre primitif ${ }^{73}$. En prenant la moyenne des gages d'un ouvrier, soit $2 \mathrm{sh}$. [ $21.8 \mathrm{~s}$.] par jour, le prélèvement représenterait de deux et demi à cinq semaines de travail et plus encore pour le journalier. Mais comme la plupart sont des producteurs indépendants, le rapport entre la rente et la valeur locative des propriétés, à partir du jumelage du rôle d'évaluation de 1821 avec la liste des débiteurs de John Mure, offre un meilleur instrument de mesure ${ }^{74}$. Lorsque l'évaluation se situe à $£ 5$ ou $£ 6-$ le tiers des propriétaires tombent dans cette catégorie - la rente du terrain correspond presque à la moitié du revenu qu'ils tirent ou pourraient tirer de leur immeuble. Et ne perdons pas de vue que cette dette s'ajoute à celle contractée pour construire ou acheter la maison, qu'il faut rembourser à court terme.

Ce n'est pas par goût que ces ouvriers engagent ainsi leurs revenus à venir pour une propriété illusoire. Ils n'ont pas d'autres choix. Les íogements à lioyer dans la ville, transformés en véritables dortoirs pour les immigrants britanniques, sont chers et inadéquats pour la famille et l'atelier. Il est impossible de construire sur un terrain non arrenté, car à l'époque les hypothèques sont à court terme, trois ans au plus, et ne couvrent pas plus de 30 à $40 \%$ de la valeur de la propriété. Ces terrains de Saint-Roch ou d'ailleurs, vendus au double du prix courant, offrent la seule solution. L'important c'est de ne pas avoir à rembourser le principal et, si les temps sont bons, ces petits producteurs peuvent sans doute joindre les deux bouts, en misant sur la main-d'oeuvre familiale, en prenant des pensionnaires, en gardant un porc ou deux dans la cour.

La rente et l'ensemble des contraintes qui l'accompagnent font partie de l'ordre des choses et, bien que nous n'ayons pas étudié cet aspect de la question, il n'y a pas lieu de penser que les habitants pouvaient se soulever contre un système que leur père et leur grand-père avaient subi avant eux. Non pas que le faubourg soit soumis et muet. Il bouge, il effraie même, mais il faut des nouveautés pour soulever sa colère. Le gouvernement en fait l'expérience quand il veut subitement, en 1834, récupérer tous les arrérages de lods et ventes dus au Domaine. Une pétition de 580 noms, apportée chez le gouverneur par une députation imposante, et plus

73 Voir le tableau 4, supra.

74 Liste détaillée du 17 août 1825, Papiers Ross, Archives du Musée McCord. 
encore l'atmosphère de mécontentement et d'excitation qui règne dans le quartier, obligent les autorités à composer avec les censitaires $^{75}$. Ce sont des années difficiles. La crise commerciale, qui frappe durement les exportations vers l'Angleterre et la construction navale, crée du chômage et le mouvement révolutionnaire qui secoue le Canada en 1837 et 1838 trouve à Saint-Roch un terreau favorable. A la suite de l'assemblée populaire tumultueuse du 25 septembre 1838, la rumeur circule à Québec que les gens du faubourg vont monter étrangler les bourgeois dans leurs lits. La découverte de trois barils de poudre et quatre-vingt livres de balles alimente ces frayeurs et pendant un certain temps la ville ferme prudemment ses portes, nuit et jour ${ }^{76}$.

Il faudrait davantage que ces quelques notes superficielles pour donner une idée juste des conditions matérielles et du climat social dans Saint-Roch, et nous revenons à ces bourgeois qui sont au point de départ de cette étude. À l'époque où Henri Hiché commence à lotir sa terre, la rente est le mode normal de placement des épargnes. Durant les soixante-quinze ans qui suivent, le pays connaît des transformations économiques importantes, augmentation de la population, essor du commerce extérieur, mais elles ne bouleversent pas encore les bases de la production. L'épargne continue donc d'être canalisée vers les formes traditionnelles de propriété, tels les droits seigneuriaux en milieu rural et ce type de «rançonnement au deuxième degré» que sont les rentes de monopole, superposées au prélèvement seigneurial ordinairement faible en milieu urbain et qui empruntent à la seigneurie ses privilèges juridiques ${ }^{77}$. Les principaux rentiers de Saint-Roch ne sont pas des petits bourgeois à mentalité retardataire mais comptent parmi les grands négociants-entrepreneurs de l'époque. L'âge mûr venant, lorsqu'il faut mettre à l'abri les profits accumulés dans le commerce, ils ne peuvent se tourner que vers la propriété et celle qui est

75 Pétition de décembre 1836: APC, MG11, série Q, vol. 236, I, $102-112$ et la suite dans le même volume, partie I et II, passim. Voir aussi les numéros du 28 décembre 1836 et de janvier 1837 du journal Le Canadien. L'article de G. Baillargeon sur cette affaire est assez déroutant car il n'aborde pas l'aspect socio-économique: «Les arrérages de lods et ventes à Québec en 1832», dans RHAF, 19,2 (septembre 1965): 296-301.

76 Voir les informations dans les journaux de Québec, entre autres, Le Canadien, 9, 12 et 23 novembre 1838; et Fernand Ouellet, Le Bas-Canada, 1791-1840(Ottawa, 1976), 475.

77 Sur le problème de la rente dans les formations urbaines pré-capitalistes et le processus de transformation des immeubles en marchandise, voir Christian Topalov, Capital et propriété foncière. Introduction à l'étude des politiques foncières urbaines (Paris, 1973), 15-51. 
garantie par le corpus féodal représente la forme la plus sûre de placement, sinon la plus profitable en des temps d'instabilité et de dépression. Après 1840, les bourgeois fortunés achèteront des actions et des obligations dans les banques et les entreprises publiques, mais, auparavant, l'économie canadienne n'a pas encore assez de maturité pour autoriser des placements dans des formes capitalistes de production. En somme, la répartition des actifs n'est pas affaire de goût, de préférence, mais reflète essentiellement l'éventail des prélèvements possibles sur le surproduit social à tel moment précis de l'évolution économique du pays ${ }^{78}$.

Vers la fin de la période étudiée, une série d'à-coups dans les rentrées viennent troubler la tranquillité des rentiers. Les arrérages ont grossi dangereusement pendant la crise commerciale qui à partir de 1837 affecte les grands chantiers et rebondit sur les ateliers et le petit commerce. Au moment où la situation commence à se rétablir, Saint-Roch disparaît dans un terrible incendie, le 28 mai 1845. Bien sûr, le terrain ne brûle pas, mais, face à la ruine des débiteurs et soùs la pressiôñ de l'ôpiniớn puoblique émue par ce maihneur, Pozer annonce qu'il accordera jusqu'à cinq ans de remise à ceux qui viendront reconnaître leur dette. L'apurement suit son cours lorsqu'il cède ses rentes à George Alford et les maisons rebâties en dur sur les décombres semblent, sur le moment, mieux épauler la rente que par le passé. Mais nous sommes déjà à l'orée d'une nouvelle période qui verra les profits des rentiers sombrer petit à petit dans la médiocrité. Leurs privilèges sont minés par la commutation du régime seigneurial en 1854 et la rédaction du code civil qui suit. Les revenus fixes se dévalorisent au fur et à mesure que les prix se relèvent. Enfin la prolétarisation du faubourg transforme les assises de l'exploitation du travail. Les rentes traînent peut-être encore dans les livres des petits épargnants, qui grapillent ce que le capital leur abandonne, mais elles ne signifient plus rien.

78 C'est une histoire paresseuse qui, au lieu d'étudier la logique interne des structures, place d'emblée l'explication d'un fait économique à un autre niveau, perçu comme hors de l'histoire, celui des valeurs individuelles ou collectives. L'oeuvre de Fernand Ouellet $s$ 'inscrit dans cette tradition et on en trouve une bonne illustration dans son article: «Propriété seigneuriale et groupes sociaux dans la vallée du Saint-Laurent, 1663-1840» (Revue de l'Université d'Ottawa (janvier-avril 1977): 183-213). Ici l'auteur distingue en dernier ressort deux catégories d'acheteurs de seigneuries: ceux qu'anime la recherche du profit, soit les Britanniques; ceux qui obéissent à des sentiments de vanité, de peur ou de nostalgie, soit les Canadiens. Et puisque les valeurs qui fondent le classement ne sont ni démontrées, ni même vraiment démontrables, on peut s'interroger sur l'utilité de ces sortes d'exercices pour l'avancement de notre connaissance du passé. 